\title{
A case of Plasmodium Falciparum Malaria with initial presentation of diarrhea leading to multi organ failure
}

Key words: Cerebral Malaria, Diarrhoea, IV Aretesunate.

\section{Introduction:}

P. falciparum, which is found worldwide in tropical and subtropical areas, and especially in Africa where this species predominates. Around 300 million are infected annually in Africa by malaria and 1 to 2 million will die from the disease. ${ }^{1}$ Of the 4 human parasitic species that have been identified $P$. falciparum can cause severe malaria because it multiples rapidly in the blood, and can thus cause severe anaemia. In addition, the infected parasites can clog small blood vessels. When this occurs in the brain, cerebral malaria results, a complication that can be fatal.It is not endemic to Europe, and reported cases in Europe are almost exclusively in travelers returning from malaria-endemic areas. ${ }^{2}$ The Netherlands was declared malaria free by the World Health Organization in 1970. Malaria is endemic in 13 eastern and north-eastern border belt districts of the Bangladesh with variable transmission potentials (high, moderate and low). A total of 13.25 million people are at risk of malaria inhabited in those districts. ${ }^{5}$ In 2013 the prevalence rate of malaria was found to be $0.7 \%$ in these districts. About $80 \%$ of the total cases are reported from the three Chittagong Hill Tract (CHT) districts (Rangamati, Khagrachari and Banderban) including Chittagong and the coastal district Cox's Bazar. ${ }^{5}$ The CHT districts have perennial transmission throughout the year due to the geo-physical location in the hilly, forested and the

\footnotetext{
1. Senior House Officer, GICU, United Hospital Ltd.

2. Intensivist and coordinator, GICU, Unted Hospital Ltd.

3. Lecturer, Dept of Microbiology, Ibrahim Medical College.

4. Professor and Consultant, GICU, United Hospital Ltd.

*Corresponding Author:
}

Dr. Mohammad Omar Faruq

Professor and Consultant, GICU, United Hospital Ltd.

E mail : faruqmo@yahoo.com

Cell : 01674999897 foot-hills, climate, and other favorable conditions for the vector species. ${ }^{5}$ Longer incubation periods can occur in semi-immune persons and persons taking ineffective malaria prophylaxis, but is typically less than one month. Importantly, diagnosis of $P$. falciparum malaria may be missed or delayed in patients who have malaria years after leaving a malaria-endemic area or who do not report recent visits to malaria-endemic countries. ${ }^{6}$ Initially, malaria presents with flu-like symptoms with high fever, fatigue, and body aches, with hot and cold stages. Signs and symptoms in children may be nonspecific, leading to delays in diagnosis. People also may have headache, nausea, shaking chills (rigors), sweating, and weakness. But the initial presentation can involve any system of the body like this patient. Our goal of presenting this case is to encourage doctors on completing fully detailed evaluation and to encourage them in considering any atypical presentation as Malaria if there is any possibility. Here we are presenting this case to enlighten our physicians about this atypical yet severe falciparum malaria patient.

\section{Case Study:}

A 64 years old male travelled to Ghana 45 days back where he stayed for 1 month. Thereafter he went to Netherlands to spend time with his family for a week. His family informed that he seemed completely fine during that time. Afterwards he went to Myanmar and stayed there for a week. He reported no health problems during this time also. Then he came to Rajshahi, Bangladesh, a non-endemic zone for malaria. On the second day of arrival in Rajshahi, he developed loose motion 3 to 4 times a day but had no fever or any other problems. 2 days after his initial complaints, he suddenly felt very weak and dizzy and lost consciousness. There was no associated convulsions. Following these events, he was admitted to a local hospital. There he was diagnosed as a case of septicemia. Initially, he was intubated and mechanical ventilation was started on $3^{\text {rd }}$ day as the patient was 
desaturated. He was then transferred to our hospital.

Though the initial peripheral blood film (PBF) showed only thrombocytopenia, the second PBF sample showed plasmodium falciparum malaria (Figure 1). He has no history of taking malarial prophylaxis.

During admission in ICU patient was unconscious and only responded to deep painful stimuli. His pulse was $134 \mathrm{bpm}$, BP:140/80mmhg (on inotrope support), temperature: $99^{\circ} \mathrm{F}$, Respiratory Rate:20breaths/min . His pupillary reaction was sluggish. He was dehydrated.

Oral Artemethar and lumefantrine combination (Lumertam) was started as IV anti-malarial was not available instantly. 4 tablets of Lumertam 20/120 mg was given instantly. Initial ABG revealed metabolic acidosis, which was managed accordingly. He was maintaining respiration through mechanical ventilation.

Within an hour after admission, patient developed atrial fibrillation. After excluding thrombus in heart chamber through echocardiogram, electrical cardioversion was done with 100J of DC shock and sinus rhythm was achieved. Patient's renal investigations revealed acute kidney injury(creatinine-3.89mg/dl, urea-252mg/dl, eGFR-15.68 $\mathrm{ml} / \mathrm{min} / 1.73 \mathrm{~m}^{2}$ for which one session of SLED (Sustained Low efficiency Dialysis) was given. He developed fever with sweating and the temperature recorded was $102^{\circ} \mathrm{F}$.

Next day IV Artesunate was started at $2.4 \mathrm{mg} / \mathrm{kg}$ body weight. Clinically the neurological status did not improve. His renal parameters did not show much improvement and he had no urine output. So he was started on CRRT (Continuous Renal Replacement Therapy) CVVHDF (Continuous VenovenousHaemofiltration) for 48 hours. His other blood parameters revealed deranged liver function with severe coagulation abnormality. His D-dimer level was 18100 $\mu \mathrm{g} / \mathrm{FEU}$ (ref 0-550), FDP $109 \mu \mathrm{g} / \mathrm{ml}($ ref $<5$ ), APTT - $37 \mathrm{sec}$ and PT - $17.10 \mathrm{sec}$. He also developed bilateral small retinal hemorrhage on temporal side of retina. Due to his low haemoglobin and low platelet count he received blood and platelet rich plasma transfusion. During the stay in ICU his highest recorded temperature was $102^{\circ} \mathrm{F}$, with sweating. His RBS (random blood sugar) was within normal limit.

6 days after getting the IV anti-malarial medication his consciousness level did not show any significant improvement other than few flickering movement of eye when given painful stimuli. He also developed collapse of right upper lobe of lung on $6^{\text {th }}$ day which was managed. After 7 days of admission in ICU the patient's family decided to take him abroad for further management.

\section{Discussion:}

In general, malaria should be suspected in the setting of fever (temperature $\geq 37.5^{\circ} \mathrm{C}$ ) and relevant epidemiologic exposure (residence in or travel to an area where malaria is endemic) ${ }^{1}$. In malaria-endemic areas with stable transmission and during high-transmission season in areas with seasonal malaria, malaria should be suspected in children with palmar pallor or hemoglobin concentration $<8 \mathrm{~g} / \mathrm{dl}$. Much of the presenting symptoms and signs of the disease mimic other diseases such as typhoid fever. ${ }^{3,4}$

Four species of the genus Plasmodium classically cause human malaria. Plasmodium falciparum is responsible for nearly all-severe disease. It is endemic in most malarious areas and is by far the predominant species in Africa. Plasmodium vivax is about as common as $\mathrm{P}$ falciparum, except in Africa. P vivax uncommonly causes severe disease, although this outcome may be more common than previously appreciated. Plasmodium ovale and Plasmodium malariae are much less common causes of disease, and generally do not cause severe illness. Plasmodium knowlesi, a parasite of macaque monkeys, is now recognized to cause occasional illnesses, including some severe disease, in humans in Southeast Asia. ${ }^{8}$

An acute attack of malaria typically begins with a prodrome of headache and fatigue, followed by fever. A classic malarial paroxysm includes chills, high fever, and then sweats. Patients may appear to be remarkably well between febrile episodes. Fevers are usually irregular, especially early in the illness, but without therapy may become regular, with 48-hour ( $\mathrm{P}$ vivax and $\mathrm{P}$ ovale) or 72-hour (P malariae) cycles, especially with non-falciparum disease. Headache, malaise, myalgias, arthralgias, cough, chest pain, abdominal pain, anorexia, nausea and vomiting are common. Seizures may represent simple febrile convulsions or evidence of severe neurologic disease. Physical findings may be absent or include signs of anemia, jaundice, splenomegaly, and mild hepatomegaly. Rash and lymphadenopathy are not typical in malaria, and thus suggestive of another cause of fever. ${ }^{8}$

There are relatively few reports on the frequency of diarrhea in malaria, but diarrhea attributable to malaria is thought to be more common among children and non-immune adults with hyperparasitaemia. The reported incidence of diarrhea during malaria varies from $5 \%$ to $38 \%$. The pathological changes in patients infected with malaria are very complex and involve many organs, including the small bowel. However, the causes of gastrointestinal manifestations during malaria are still not clear, and the mechanism of diarrhea during malaria is likely to be multifactorial. Massive gastrointestinal bleedings with multiple foci of mucosal hemorrhage have also been observed. Tumor necrosis factor has been implicated in malaria and free oxygen radicals which can cause tissue injury in the liver, pancreas and intestine are enhanced during malaria infection. This can result in various disorders of the digestive system including diarrhea and intestinal bleeding. Prostaglandins and cyclic AMP may also be involved in the development of diarrhea in malaria. ${ }^{7}$

In the developed world, it is imperative that all persons with suggestive symptoms, in particular fever, who have traveled in an endemic area be evaluated for malaria. The risk for falciparum malaria is greatest within two months of return from travel; other species may cause disease many months - and occasionally more than a year - after return from an endemic area. Severe malaria is characterized by signs of severe illness, organ dysfunction, or a high parasite load (peripheral parasitaemia greater than $5 \%$ or greater than 
200,000 parasites $/ \mathrm{mcl}$ ). It is principally a result of $\mathrm{P}$ falciparum infection because this species uniquely infects erythrocytes of all ages and mediates the sequestration of infected erythrocytes in small blood vessels, thereby evading clearance of infected erythrocytes by the spleen. ${ }^{8}$

Severe falciparum malaria can include dysfunction of any organ system, including neurologic abnormalities progressing to alterations in consciousness, repeated seizures, and coma (cerebral malaria); severe anemia; hypotension and shock; non-cardiogenic pulmonary edema and the acute respiratory distress syndrome; acute kidney injury due to acute tubular necrosis or, less commonly, severe hemolysis; hypoglycemia; acidosis; hemolysis with jaundice; hepatic dysfunction; retinal hemorrhages and other fundoscopic abnormalities; bleeding abnormalities, including disseminated intravascular coagulation; and secondary bacterial infections, including pneumonia and Salmonella bacteremia. Our patient had developed atrial fibrillation. However, no literature exists of malaria resulting in atrial fibrillation and we believe this may be the first reported case of malaria complicated by atrial fibrillation. In the developing world, severe malaria and deaths from the disease are mostly in young children, in particular from cerebral malaria and severe anemia. Cerebral malaria is a consequence of a single severe infection while severe anemia follows multiple malarial infections, intestinal helminthes, and nutritional deficiencies. In a large trial of African children, acidosis, impaired consciousness, convulsions, renal impairment, and underlying chronic illness were associated with poor outcome. ${ }^{8}$

Severe malaria is a medical emergency. Parenteral treatment is indicated for severe malaria, as defined above, and with inability to take oral drugs. With appropriate prompt therapy and supportive care, rapid recovery may be seen even in very ill individuals. The standard of care for severe malaria is intravenous Artesunate, which has demonstrated superior efficacy and better tolerability than quinine. The drug is administered in four doses of $2.4 \mathrm{mg} / \mathrm{kg}$ over 3 days, every 12 hours on day 1 and then daily. ${ }^{8}$

In endemic regions, if parenteral therapy is not available, per rectal administration of artemether or artesunate is also effective. Patients receiving intravenous quinine or quinidine should receive continuous cardiac monitoring; if QTc prolongation exceeds $25 \%$ of baseline, the infusion rate should be reduced. Blood glucose should be monitored every4-6 hours, and 5-10\% dextrose may be co-administered to decrease the likelihood of hypoglycemia. Appropriate care of severe malaria includes maintenance of fluids and electrolytes; respiratory and hemodynamic support; and consideration of blood transfusions, anticonvulsants, antibiotics for bacterial infections, and hemofiltration or hemodialysis. With high parasitaemia (greater than 5-10\%), exchange transfusion has been used, but beneficial effects have not clearly been demonstrated and it is generally no longer recommended. ${ }^{8}$

\section{Conclusion:}

Diarrhea is a common presentation of mostly gastrointestinal diseases which are usually not fatal. This case of malaria presented initially with only GI upset and also the initial peripheral blood film showed no malaria parasites. Clinical pattern of the fever also did not coincide with the falciparum species. This case is for awareness of the physicians so that prompt diagnosis and management is essential.

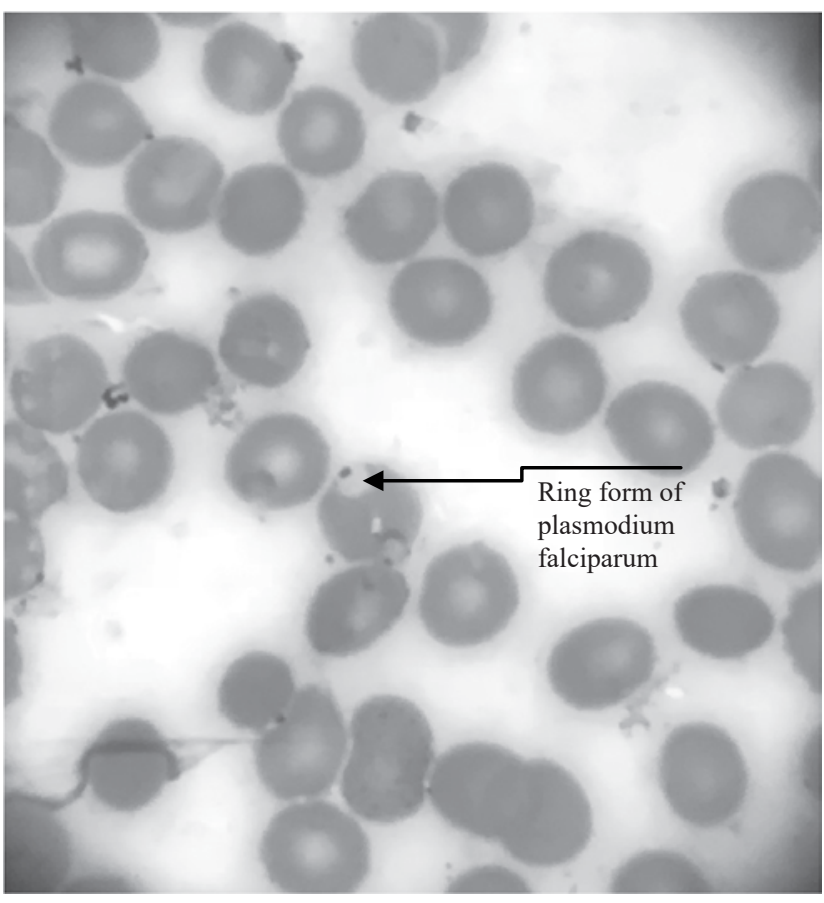

Figure 1: plasmodium falciparum

\section{References:}

1. Laishram DD, Sutton PL, Nanda N, Sharma VL, Sobti RC, Carlton $\mathrm{JM}$ et al. The complexities of malaria disease manifestations with a focus on asymptomatic malaria. Malar J. 2012; 11:29

2. Askling HH, Bruneel F, Burchard G, Castelli F, Chiodini PL, Grobusch MP et al. P. Management of imported malaria in Europe. Malar J. 2012;11:328-11

3. Brown CS, Cropley IM. Ebola virus disease: where are we now and where do we go?Postgrad Med J. 2014; 90:610-612

4. Benowitz I, Ackelsberg J, Balter SE . Surveillance and prepearedness for Ebola virus disease-New York City, 2014. Centers for Disease Control and Prevention MMWR. 2014; 63:934-936

5. The Independent 27 July 2015 http://www.theindependentbd.com/printversion/details/9123

6. Kyronseppa H, Tiula E, Repo H, Lahdevirta J. Diagnosis of falciparum malaria delayed by long incubation period and misleading presenting symptoms: life-saving role of manual leucocyte differential count. Scand J Infect Dis. 1989;21:117-118

7. Prasad RN, Virk KJ.Malaria as a cause of diarrhoea--a review. $P N$ G Med J. 1993 Dec;36(4):337-41

8. Chatterjee K. D. Parasitology (Protozoology and Helminthology). $13^{\text {th }}$ ed. New Delhi (India): CBS Publishers. 2009. Chapter 3, Phylum Apicomplexa; $\mathrm{p}$ 90-127. 